\section{Preserving the curve evolution property for the binary level set method}

\author{
Guopu Zhu, ${ }^{a}$ Shuqun Zhang, ${ }^{\text {b }}$ Qingshuang Zeng, ${ }^{\text {a }}$ and \\ Changhong Wang ${ }^{\mathrm{a}}$ \\ ${ }^{a}$ Harbin Institute of Technology, Space Control and \\ Inertial Technology Research Center, Harbin, China \\ E-mail: zgp@hit.edu.cn \\ ${ }^{\mathrm{b}}$ City University of New York, Department of Computer \\ Science, College of Staten Island, Staten Island, \\ New York 10314
}

\begin{abstract}
We present a modified binary level set method for twophase image segmentation, which is based on the binary level set method originally proposed by X.-C. Tai et al. [Int. J. Comput. Vis. 73, 61-76 (2007)]. The modified binary level set method is superior to Tai et al.'s binary level set method in preserving the curve evolution property of gradually shrinking and expanding the partition interface represented by a binary level set function in the segmentation process. Some experiments conducted on real images show the good results of the modified binary level set method. () 2007 SPIE and IS\&T. [DOI: 10.1117/1.2731338]
\end{abstract}

\section{Introduction}

The level set method, initially mentioned in the area of fluid dynamics, ${ }^{1,2}$ was recently introduced by Osher and Sethian $^{3}$ as a numerical device for capturing the interface that partitions a domain into several subdomains. Although the level set method has been successfully applied in image segmentation, ${ }^{4-8}$ its numerical implementation is computationally expensive and thus has excluded itself from realtime applications. The expensive computational cost is mainly due to the need to periodically reinitializing the level set function to a signed distance function as well as other factors such as dense level set grids and the upwind finite difference scheme. To reduce the computational cost of the traditional level set method, some techniques such as the binary level set methods ${ }^{9-11}$ and discrete level set implementation ${ }^{12}$ have been recently proposed. The binary level set methods ${ }^{9-11}$ employ a binary level set function that uses the values of 1 and -1 to represent the object and background regions, respectively. They can efficiently perform image segmentation, but tend to lose the curve evolution property of gradually shrinking and expanding the partition interface represented by the binary level set function in the segmentation process. In this letter, based on Tai et al.'s binary level set method, ${ }^{11}$ we present a modified binary level set method for two-phase image segmentation, which can preserve the curve evolution property well and maintain the computational efficiency of Tai et al.'s binary level set method.

Paper 06208LR received Nov. 26, 2006; revised manuscript received Feb. 13, 2007; accepted for publication Feb. 23, 2007; published online Apr. 24, 2007.

$1017-9909 / 2007 / 16(2) / 020502 / 3 / \$ 25.00$ @ 2007 SPIE and IS\&T.

\section{Problem Description}

Given a two-dimensional gray-value image $u_{0}: \Omega \rightarrow R^{+}$, which consists of two distinct regions $\Omega_{1}$ and $\Omega_{2}$, we represent these two regions by a binary level set function $\phi$ that takes 1 in $\Omega_{1}$ and -1 in $\Omega_{2}$. Assuming that the image $u_{0}$ can be approximated by a binary function defined by

$u\left(\phi, c_{1}, c_{2}\right)=\frac{c_{1}}{2}(1+\phi)+\frac{c_{2}}{2}(1-\phi)$,

where $c_{1}$ and $c_{2}$ are two nonnegative constants, then the problem of partitioning $\Omega$ into $\Omega_{1}$ and $\Omega_{2}$ can be modeled as minimizing the following energy functional ${ }^{9-11}$

$F\left(\phi, c_{1}, c_{2}\right)=\frac{1}{2} \int_{\Omega}\left|u\left(\phi, c_{1}, c_{2}\right)-u_{0}\right|^{2} \mathrm{~d} x+\beta \int_{\Omega}|\nabla \phi| \mathrm{d} x$,

subject to

$\phi^{2}=1$.

The first term in the right-hand side of Eq. (2) measures how well the binary function $u$ approximates $u_{0}$. The second term measures the length of the partition interface represented by the binary level set function $\phi . \beta$ is a weighting parameter that controls the length regularization.

\section{The Proposed Method}

Because directly solving the constrained minimization problem formulated by Eqs. (2) and (3) is somewhat difficult, Tai et al. have proposed an efficient binary level set method to obtain the approximate solution of the problem. ${ }^{11}$ Their binary level set method is an iterative process. In each iteration, the energy functional $F$ in Eq. (2) is first optimized without considering the restriction of Eq. (3). The associated Euler-Lagrange equation of the energy functional $F$ can be implemented by the following gradient descent:

$\frac{\mathrm{d} \phi}{\mathrm{d} t}=\beta \boldsymbol{\nabla} \cdot\left(\frac{\boldsymbol{\nabla} \phi}{|\boldsymbol{\nabla} \phi|}\right)-\left[u\left(\phi, c_{1}, c_{2}\right)-u_{0}\right] \frac{\partial u}{\partial \phi}$,

where the constants $c_{1}$ and $c_{2}$ in Eq. (4) are given by

$c_{1}=\frac{\int_{\Omega} u_{0}(1+\phi) \mathrm{d} x}{\int_{\Omega}(1+\phi) \mathrm{d} x}, \quad c_{2}=\frac{\int_{\Omega} u_{0}(1-\phi) \mathrm{d} x}{\int_{\Omega}(1-\phi) \mathrm{d} x}$.

Then, the function $\phi$ is set to be a binary level set function compulsively, that is, let $\phi$ take 1 if $\phi \geq 0$, otherwise let $\phi$ take -1 .

Unfortunately, in Tai et al.'s binary level set method ${ }^{11}$ and their related work, ${ }^{9,10}$ the sign of the binary level set function $\phi(x)$ tends to vary without a priority at any location $x$ when the level set function $\phi$ evolves. This makes the binary level set method lose the curve evolution property of gradually shrinking and expanding the partition interface represented by the level set function in the segmentation process. To preserve the curve evolution property, the sign variation of the binary level set function value should 


\section{J E I L E T T E R S}

be restricted to the neighborhood of the partition interface when the binary level set function evolves. Therefore, we modify Eq. (4) as

$$
\frac{\mathrm{d} \phi}{\mathrm{d} t}=\left|G_{\sigma} * \boldsymbol{\nabla} \phi\right|\left\{\beta \boldsymbol{\nabla} \cdot\left(\frac{\boldsymbol{\nabla} \phi}{|\boldsymbol{\nabla} \phi|}\right)-\left[u\left(\phi, c_{1}, c_{2}\right)-u_{0}\right] \frac{\partial u}{\partial \phi}\right\},
$$

where $G_{\sigma}$ denotes a Gaussian filter with standard deviation $\sigma$. For the binary level set function $\phi$, the new term $\left|G_{\sigma}{ }^{*} \nabla \phi\right|$ will be approximately 0 when the location $x$ is far away from the partition interface; whereas, it will have relatively large value when the location $x$ is close to the partition interface. Therefore, the added new term has the effect of restraining the variation of the level set function $\phi$ except in the neighborhood of the partition interface. In other words, the effect of the new term helps to preserve the curve evolution property for the binary level set method.

Based on the preceding description, we present the modified binary level set method as follows:

1. Initialize the binary level set function $\phi$.

2. Compute the constants $c_{1}$ and $c_{2}$ according to Eq. (5).

3. Evolve the level set function $\phi$ according to Eq. (6).

4. Let $\phi$ take 1 if $\phi \geq 0$, otherwise let $\phi$ take -1 . Go to Step 2 .

The preceding process proceeds until the evolution of the binary level set function has converged. It is noted that if we replace Eq. (6) with Eq. (4) in Step 3, we can obtain Tai et al.'s binary level set method on two-phase image segmentation.

\section{Experimental Results}

We conducted several experiments to show the performance of the modified binary level set method. Figure 1 shows the partition interface evolution of the modified binary level set method on segmenting a synthetic image, which indicates that the modified binary level set method can effectively preserve the curve evolution property. In Fig. 2, we use two examples to compare the performance between Tai et al.'s binary level set method and the modified binary level set method on real image segmentation. Each of the real images is $240 \times 240$ pixels in size. $\beta$ $=0.002 \times 255^{2}$ was used for both methods and $\sigma=2$ was used for the Gaussian filter $G_{\sigma}$ in the modified method. The upper row of Fig. 2 shows the segmentation results of the cup image, respectively, using Tai et al.'s binary level set method and the modified binary level set method. Due to losing the curve evolution property mentioned above, Tai et al.'s binary level set method performs the image segmentation like an adaptive threshold segmentation method that considers the spatial constrains. Therefore the segmented object region includes the white regions belonging to the background, but excludes the dark regions inside the object boundary, as shown in Fig. 2(b). On the contrary, the partition interface of the modified binary level set method can exactly converge to the cup boundary from the initial position. The lower row of Fig. 2 shows the segmentation results of the corpus-callosum magnetic resonance (MR) im-

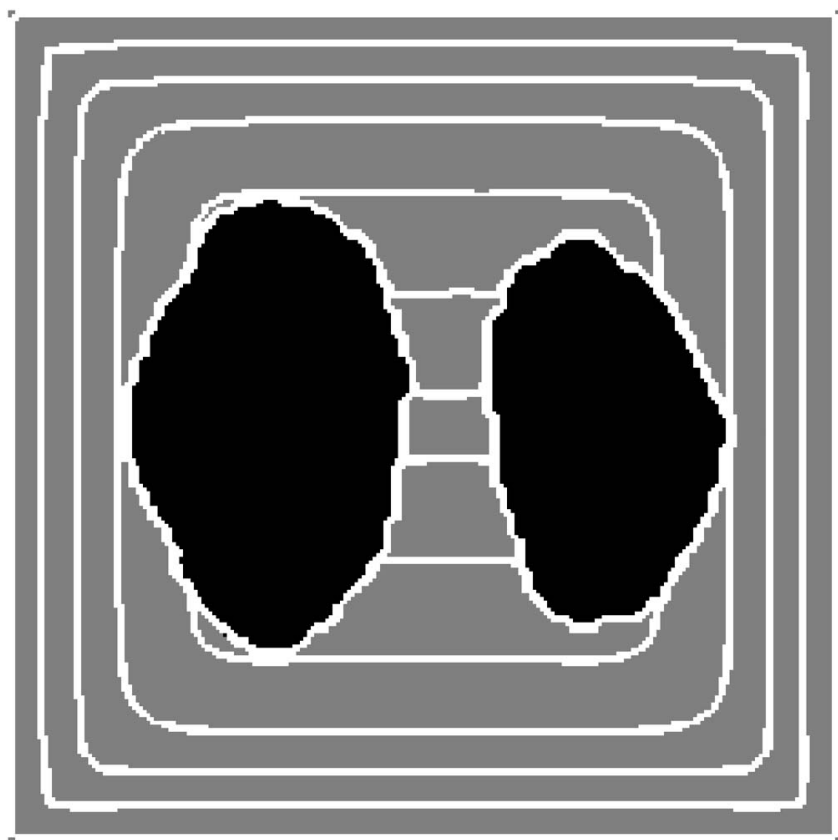

Fig. 1 Evolution of the partition interface in segmenting a synthetic image using the modified binary level set method.

age. It is seen that the modified binary level set method also outperforms Tai et al.'s binary level set method.

\section{Conclusion}

In this Letter, we present a modified binary level set method for two-phase image segmentation, which has the advantage of preserving the curve evolution property over the original binary level set method proposed by Tai et al.

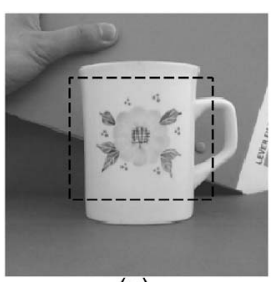

(a)

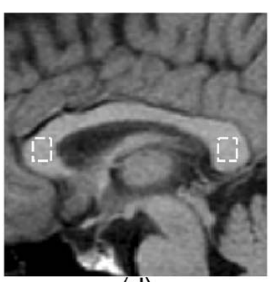

(d)

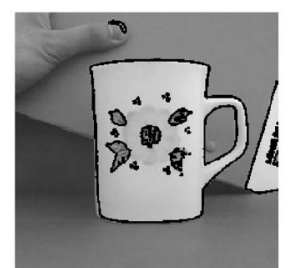

(b)

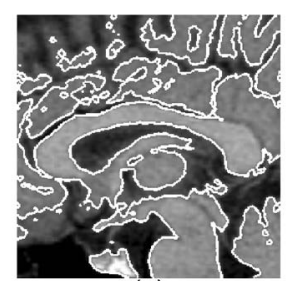

(e)

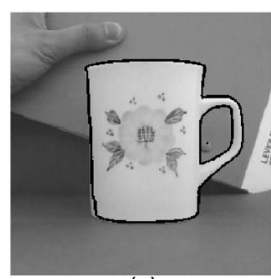

(c)

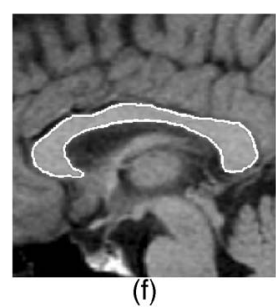

Fig. 2 Tests on segmenting real images: (a) the original cup image with the initial interface, (b) and (c) the segmentation results of the cup image using Tai et al.'s binary level set method and the modified binary level set method, respectively; (d) the original corpuscallosum MR image with the initial interface, (e) and (f) the segmentation results of the corpus-callosum MR image using Tai et al.'s binary level set method and the modified binary level set method, respectively. Note that the dashed lines represent the initial partition interfaces, whereas the solid lines represent the partition interfaces at the steady states. 


\section{J E I L E T T E R S}

The experiments demonstrate the capability of the proposed binary level set method. In the future, we will extend the modified binary level set method to multi-phase image segmentation. ${ }^{7,9-11}$

\section{Acknowledgment}

The authors would like to thank the anonymous reviewers for their valuable comments and suggestions.

\section{References}

1. A. Dervieux and F. Thomasset, "A finite element method for the simulation of Rayleigh-Taylor instability," Lect. Notes Math. 771, 145-159 (1979).

2. A. Dervieux and F. Thomasset, "Multifluid incompressible flows by a finite element method," Lect. Notes Phys. 141, 158-163 (1981).

3. S. Osher and J. A. Sethian, "Fronts propagating with curvature dependent speed: Algorithms based on Hamilton-Jacobi formulations," J. Comput. Phys. 79, 12-49 (1988).
4. V. Caselles, F. Catté, C. Coll, and F. Dibos, "A geometric model for active contours in image processing," Numer. Math. 66, 1-31 (1993),

5. V. Caselles, R. Kimmel, and G. Sapiro, "Geodesic active contours," Int. J. Comput. Vis. 22, 61-79 (1997).

6. T. F. Chan and L. A. Vese, "Active contours without edges," IEEE Trans. Image Process. 10, 266-277 (2001).

7. L. A. Vese and T. F. Chan, "A multiphase level set framework for image segmentation using the Mumford and Shah model," Int. J. Comput. Vis. 50, 271-293 (2002).

8. G. Zhu, Q. Zeng, and C. Wang, "Dual geometric active contour for image segmentation," Opt. Eng. 45, 080505 (2006).

9. J. Lie, M. Lysaker, and X.-C. Tai, "A variant of the level set method and applications to image segmentation," Math. Comput. 75, 11551174 (2006).

10. J. Lie, M. Lysaker, and X.-C. Tai, "A binary level set model and some applications to Mumford-Shah image segmentation," IEEE Trans. Image Process. 15, 1171-1181 (2006)

11. X.-C. Tai, O. Christiansen, P. Lin, and I. Skjælaaen, "Image segmentation using some piecewise constant level set methods with MBO type of project," Int. J. Comput. Vis. 73, 61-76 (2007).

12. Y. Shi and W. C. Karl, "A fast level set method without solving PDEs," Proc. IEEE Int. Conf. ICASSP 2, 97-100 (2005). 